\title{
Informe de autoevaluación como herramienta formativa para árbitros de fútbol. Estudio de caso
}

\author{
Self-assessment report as a formative tool for football referees. A case study \\ Informe d'autoavaluació com a eina formativa per a àrbitres de futbol. Estudi de cas
}

\author{
David Recio-Moreno*® $^{*}$, Tiberio Feliz-Murias ${ }^{\circledR}$ \\ Departamento de Didáctica, Organización Escolar y Didácticas Especiales. Facultad de Educación, \\ Universidad Nacional a Distancia, Madrid, España \\ *Autor para correspondencia: davidrecio@bec.uned.es (David Recio-Moreno)
}

Recibido: 26/05/2020 | Aceptado: 27/10/2020 | Publicado: 24/12/2020

Cómo citar: Recio-Moreno, D. y Feliz-Murias, T. (2020). Informe de autoevaluación como herramienta formativa para árbitros de fútbol. Estudio de caso. Research in Education and Learning Innovation Archives, 25,33-53.

10.7203/realia.25.17453

Copyright: El/La Autor/a. Open Access: Este es un artículo de acceso abierto distribuido bajo los términos de la licencia Creative Commons

Attribution-NoDerivatives 4.0 International licence (CC BY-ND 4.0)

\section{Financiación: None} informed
RESUMEN: Este artículo ofrece los resultados de una investigación evaluativa centrada en una práctica educativa que se desarrolló durante un torneo internacional de fútbol y que puso el foco en la formación de los árbitros participantes. La experiencia tuvo el objetivo de facilitar a los árbitros un instrumento de autoevaluación para analizar y valorar sus actuaciones arbitrales en los encuentros que dirigieron durante el campeonato. Esta herramienta pretendía, además, servir al equipo docente como fuente de información para detectar las necesidades del grupo en cada jornada y facilitar procesos de feedback dialógico en el marco de una evaluación formativa. Esta retroalimentación se llevó a cabo en las distintas sesiones de formación, caracterizadas por una metodología participativa, interactiva y colaborativa, valorando el vídeo como un recurso didáctico fundamental. Este estudio de caso se desarrolló desde una complementariedad metodológica, cualitativa y cuantitativa, aplicando diversas técnicas e instrumentos. Se analizó el contenido de un conjunto de 371 informes de autoevaluación, elaborados tras cada partido por el grupo de 28 árbitros participantes en el torneo. Se investigó la utilidad y las aportaciones de la herramienta de autoevaluación, así como el nivel de satisfacción con la propuesta de formación, a través del análisis de los resultados de un cuestionario que se administró a los árbitros y de un grupo de discusión con los formadores. Los resultados evidenciaron un alto grado de satisfacción y aceptación del instrumento y de la metodología desarrollada en las reuniones de trabajo. Destacan el valor del vídeo como material didáctico, la calidad de la retroalimentación recibida y del enfoque participativo, interactivo y colaborativo de las diferentes sesiones. Esta propuesta pretende ser transferible a otras situaciones o contextos formativos.

PALABRAS CLAVE: formación permanente; retroalimentación formativa; autoevaluación formativa; árbitros de fútbol

ABSTRACT: In this article we present the results of an evaluative study of an educational practice that focused on the training of participating referees at an international football tournament. The aim was to provide referees with a self-assessment instrument for analyzing and evaluating their refereeing performances at the tournament. The instrument also provided the teaching team with a source of information for detecting the referees' daily needs and facilitating dialogical feedback processes in the context of a formative evaluation. Feedback was conducted at training meetings characterized by a participative, interactive and collaborative methodology in which video was used as a fundamental didactic resource. This case study employed a range of complementary methodological, qualitative and quantitative techniques and instruments. We analyzed 371 self-assessment 
reports produced after the matches by the 28 participating referees and evaluated the usefulness and contributions of the self-assessment tool. We also evaluated the referees' level of satisfaction with the training proposal by analyzing their responses to a questionnaire and holding discussions with their trainers. The results revealed a high level of satisfaction and acceptance of both the instrument and the methodology developed at the work meetings. They also highlight the value of video content as teaching material, the quality of the feedback received, and the participatory, interactive and collaborative approach adopted at the sessions. This proposal is intended to be transferable to other situations and training contexts.

KEYWORDS: permanent education; formative feedback; formative self-assessment; soccer referees

RESUM: Aquest article ofereix els resultats d'una investigació avaluativa centrada en una pràctica educativa que es va desenvolupar durant un torneig internacional de futbol i que va posar el centre d'atenció en la formació dels àrbitres participants. L'experiència tenia l'objectiu de facilitar als àrbitres un instrument d'autoavaluació per analitzar i valorar les seues actuacions arbitrals en els partits que van dirigir durant el campionat. Aquesta eina pretenia, a més, servir a l'equip docent com a font d'informació per detectar les necessitats del grup en cada jornada i facilitar processos de feedback dialògic en el marc d'una avaluació formativa. Aquesta retroalimentació es va dur a terme en les diferents sessions de formació, caracteritzades per una metodologia participativa, interactiva i col-laborativa, amb una valoració del vídeo com un recurs didàctic fonamental. Aquest estudi de cas es va desenvolupar des d'una complementarietat metodològica, qualitativa i quantitativa, amb aplicació de diverses tècniques i instruments. Es va analitzar el contingut d'un conjunt de 371 informes d'autoavaluació, elaborats després de cada partit pel grup de 28 àrbitres participants en el torneig. Es va investigar la utilitat i les aportacions de l'eina d'autoavaluació, així com el nivell de satisfacció amb la proposta de formació, a través de l'anàlisi dels resultats d'un qüestionari que es va administrar als àrbitres i d'un grup de discussió amb els formadors. Els resultats van evidenciar un alt grau de satisfacció i acceptació de l'instrument i de la metodologia desenvolupada en les reunions de treball. Destaquen el valor del vídeo com a material didàctic, la qualitat de la retroalimentació rebuda i de l'enfocament participatiu, interactiu i col-laboratiu de les diferents sessions. Aquesta proposta pretén ser transferible a altres situacions o contextos formatius.

PARAULES CLAU: formació permanent; retroalimentació formativa; autoavaluació formativa; àrbitres de futbol

\section{Notas de aplicación práctica}

\section{Qué se sabe del tema}

- Son escasos los trabajos de investigación cuyo objeto de estudio se ha centrado en el área de formación de árbitros y asistentes de fútbol. En este manuscrito se recogen aquellas aportaciones más relevantes que contribuyen a la mejora de su actividad en los actuales contextos de formación arbitral.

\section{Qué aporta este trabajo}

- Este manuscrito muestra un instrumento de autoevaluación, dirigido a los árbitros, para analizar y valorar la actuación arbitral después de un partido de fútbol. Esta herramienta se presenta como un recurso valioso para el equipo docente ya que permite obtener información útil para detectar las necesidades y áreas de mejora, y facilitar procesos de feedback dialógico en el en marco de una propuesta formativa que viene caracterizada por una metodología participativa, interactiva y colaborativa.

\section{Implicaciones para la práctica y / o política}

- Los resultados obtenidos pueden suponer una importante fuente de información para los formadores de árbitros y asistentes de fútbol que tengan interés en diseñar y desarrollar experiencias formativas con este colectivo en otros contextos, valorando el instrumento de autoevaluación y su enfoque pedagógico. 


\section{INTRODUCCIÓN Y ESTADO DE LA CUESTIÓN}

En el mundo del fútbol, la figura del árbitro adquiere un papel fundamental ya que es la persona encargada de controlar un partido, en colaboración del resto de miembros del equipo arbitral (árbitros asistentes, cuarto árbitro, entre otros), y de hacer cumplir las reglas de juego (IFAB, 2019; Samuel, Galily, y Tenenbaum, 2017). Alcanzar un nivel elevado en el plano físico, técnico, táctico y psicológico es esencial (González-Oya, 2005) para tomar decisiones correctas ante las situaciones que se presentan durante el juego en los partidos dirigidos en una competición. En consecuencia, este colectivo experimenta cambios constantes para adaptarse a la evolución de este deporte y desarrollar su compleja actividad con éxito (Dosseville y Laborde, 2015).

En el contexto de la formación arbitral, la dimensión técnica y táctica se encuentran estrechamente relacionadas y tienen un peso relevante. La primera se vincula con el conocimiento, la aplicación e interpretación de las reglas de juego. Mientras que la segunda aborda el conjunto de recursos y herramientas que utiliza el árbitro para controlar y gestionar un partido, interactuar con el resto de participantes, analizar y evaluar las situaciones de juego, etc. Por ello, la toma de decisiones del árbitro en un partido es una de las tareas clave en el desempeño de su actividad dentro del terreno de juego (Cardoso, Travassos, y Oliveira, 2020; Hancock, Rix-Lièvre, y Côté, 2015).

Durante los últimos años, se han llevado a cabo investigaciones que ponen el foco en los procesos de enseñanza y de aprendizaje de los árbitros y asistentes de fútbol. Sin embargo, la producción científica en esta parcela no es abundante. Estos estudios se centran en distintos elementos del proceso formativo cuyo objetivo es aportar líneas de trabajo y de mejora que contribuyan al progreso de su actividad deportiva, ajustándose de mejor manera a las exigencias que cada campeonato o competición demanda.

Entre ellos, Armenteros y Benítez (2011) presentan el vídeo test interactivo. Este modelo de evaluación muestra vídeos con distintas situaciones reales de juego, donde el usuario selecciona la decisión técnica y la sanción disciplinaria correcta. Del mismo modo, este material interactivo se implementa con árbitros asistentes para juzgar situaciones específicas de fuera de juego (Armenteros et al., 2018; Armenteros, Benítez, y Sillero, 2009) mantienen este modelo de autoevaluación como un recurso útil en el proceso formativo de árbitros, asistentes e instructores. Destacan la potencialidad didáctica de los materiales multimedia al favorecer el análisis de jugadas reales y su uso en sesiones formativas para fomentar la participación e interacción de los usuarios.

En esta misma línea, Armenteros, Benítez, y Curca $(2011,2013)$ muestran un modelo de juego interactivo basado en cuestionarios de preguntas tipo test sobre el contenido de las reglas de juego. Plantean esta opción como un recurso útil y relevante para el aprendizaje y la evaluación de las reglas de juego. Además, (Armenteros, Domínguez, Fernández, y Benítez, 2016) presentan la herramienta de base de datos de vídeo utilizada por la Fédération Internationale de Football Association (FIFA) en sus cursos y seminarios de formación arbitral. Este recurso educativo se caracteriza por contener vídeos sobre situaciones de juego de diferentes competiciones y campeonatos, clasificados por temáticas relacionadas con las reglas de juego, las decisiones técnicas, las sanciones disciplinarias y sus consideraciones.

Por todo ello, el colectivo arbitral se convierte en una interesante fuente de estudio para analizar su realidad y proceso de formación continua. Surge la necesidad de conocer las oportunidades que ofrecen los distintos enfoques metodológicos basados en la participación, interacción y colaboración (Armenteros et al., 2019; Recio-Moreno, Feliz-Murias, y Elorza-Barbajero, 2020), las prestaciones de la tecnología, el desarrollo de nuevas herramientas y materiales didácticos multimedia (Armenteros, Liaw, Fernández, Flores-Díaz, y Arteaga-Sánchez, 2013) (Armenteros, Liaw, Sánchez-Franco, Fernández, y Arteaga-Sánchez, 2017), con el objetivo final de abordar el diseño de escenarios y situaciones de aprendizaje que contribuyan a la mejora de su formación permanente. 
Así pues, este artículo presenta el escenario que surge del desarrollo de una acción formativa dirigida a los árbitros participantes en un torneo internacional de fútbol. La práctica se basa en un enfoque metodológico participativo, interactivo y colaborativo. El dispositivo móvil, el vídeo y el informe de autoevaluación se convierten en medios y recursos fundamentales en este proceso formativo. Por un lado, ofrece a los árbitros participantes un instrumento de autoevaluación para analizar y valorar sus actuaciones en los encuentros dirigidos en el campeonato. Por otro lado, esta herramienta proporciona a los formadores una fuente de información relevante para conocer el desempeño de las actuaciones y para detectar las necesidades del grupo en cada jornada, ayudando a facilitar procesos de feedback en las sesiones formativas.

En relación a esta propuesta, distintos autores (Cano, 2014; Carless, 2012, 2015; Nicol, 2011) consideran que la evaluación y el feedback tienen un papel importante en los procesos de enseñanza y aprendizaje. Esta acción educativa surge de la necesidad de aportar herramientas de autoevaluación a los árbitros de fútbol para facilitar el análisis y valoración de su actividad en sus partidos. Esto permite detectar los aspectos con recorrido de mejora para ser trabajados en las sesiones de formación desde un enfoque interactivo, participativo y colaborativo. Así, se aborda el feedback como estrategia que favorece el diálogo entre los participantes del proceso formativo.

Desde esta perspectiva, la evaluación y el feedback facilitado en los procesos educativos son elementos clave que nos permiten situarnos en el qué, cómo y cuándo aprende el alumnado (Orsmond, Maw, Park, Gomez, y Crook, 2013). Gracias al feedback, el formador guía y acompaña el proceso de aprendizaje del alumnado, facilitando ayudas y orientaciones que permitan reflexionar sobre el proceso y sobre cómo mejorar el desempeño en futuras actividades (Nicol, 2011), promoviendo progresivamente la autorregulación de su aprendizaje. El feedback debe ser contextualizado, es decir, debe considerar los objetivos de la actividad que se está desarrollando. El alumnado se muestra más receptivo al feedback cuando comprenden la finalidad que acompaña la propuesta (Price, Handley, Millar, y O’Donovan, 2010). En relación con este aspecto, Panadero y Romero (2014) sostienen que el uso de rúbricas ayuda a clarificar y compartir los objetivos y criterios de evaluación entre los distintos actores. Este elemento ayuda al proceso de feedback elaborado por el formador (Panadero, AlonsoTapia, y Huertas, 2012) y apoya el proceso de autorregulación (Panadero y Jonsson, 2013).

El feedback proporcionado al alumnado trata de provocar retos alcanzables y alienta a una mejora continua. Debe ser adecuado en relación al tiempo y al contenido de la actividad realizada. Hace referencia a los resultados esperados y a los criterios de evaluación. Por último, señala los aspectos positivos y con recorrido de mejora para ofrecer información y herramientas para mejorar los desempeños posteriores.

Por otro lado, (Nicol, 2007) afirma la relevancia de que el proceso de feedback sea también una ayuda para los formadores ya que les permite adaptar y flexibilizar la enseñanza a las necesidades detectadas del alumnado, permitiéndoles reflexionar sobre la manera de enfocar la manera de abordar las sesiones de trabajo.

Siguiendo a Nicol (2011), la implicación del alumnado hacia la comprensión y el uso del feedback es importante, así como la calidad del feedback proporcionado por el formador. Algunos estudios indican que, en muchas ocasiones, el proceso de feedback tiene una eficacia limitada ya que el alumnado no utiliza ni comprende el feedback (Gibbs y Simpson, 2004; Nicol, 2011; Price et al., 2010). Sin embargo, la comprensión, el uso del feedback y su compromiso, no recae únicamente en la responsabilidad del alumnado. Orsmond y Merry (2011) sostienen la importancia de orientar al alumnado sobre el uso del feedback. Por ello, Quinton y Smallbone (2010) hacen hincapié en el diseño de los procesos de enseñanza y aprendizaje, de espacios y tiempos para la reflexión del feedback. En esta línea, Nicol (2011) señala que una manera de obtener mayor impacto del feedback en el alumnado está en generar oportunidades para la 
participación en los encuentros, conversaciones y diálogos de feedback de manera conjunta con el formador y con el resto de los compañeros, y reflexionar así sobre el mismo, es decir, promover el llamado feedback dialógico. El diálogo entre los actores es importante para la mejora de la comprensión del feedback y del aprendizaje a partir del mismo (Hattie y Timperley, 2007; Liu y Carless, 2006; Nicol, 2010; Nicol y MacfarlaneDick, 2006).

Por último, formalmente, esta investigación busca alcanzar los siguientes objetivos:

- Describir y comprender de qué forma el instrumento de autoevaluación tiene un impacto en el proceso educativo de los árbitros de fútbol y es un medio útil en acciones formativas participativas, interactivas y colaborativas.

- Determinar la experiencia de los árbitros participantes al utilizar el instrumento de autoevaluación como recurso para analizar y valorar el desempeño de sus actuaciones arbitrales en los partidos dirigidos durante el campeonato.

- Conocer la opinión de los formadores sobre la utilidad y las aportaciones del instrumento de autoevaluación como fuente de información para conocer el desempeño y detectar las necesidades de los árbitros en el torneo.

- Abordar el desempeño de las actuaciones arbitrales a través del análisis de los informes de autoevaluación para detectar las necesidades formativas del grupo.

\section{MÉTODO}

\subsection{Diseño metodológico}

Para alcanzar los objetivos propuestos, se planteó un diseño de investigación mixta de estudio de caso (Stake, 2005), desde una complementariedad metodológica, cuantitativa y cualitativa. Se analizó la práctica pedagógica desarrollada y las opiniones de los participantes y docentes respecto a las estrategias metodológicas aplicadas y la integración del informe de autoevaluación como medio formativo. Por un lado, se analizó en contenido de un conjunto de 371 de informes de autoevaluación (desempeño en las actuaciones arbitrales en cada dimensión del informe; necesidades formativas detectadas del grupo en cada una de las jornadas), elaborados por los árbitros participantes en la experiencia educativa en cada uno de los partidos dirigidos en el torneo. Por otro lado, se administró un cuestionario para conocer el nivel de satisfacción de los árbitros sobre el instrumento de autoevaluación utilizado y sobre la acción formativa recibida. Por último, se desarrolló un grupo de discusión con los instructores arbitrales que participaron en la práctica formativa para explorar su opinión acerca de la utilidad y de las aportaciones del instrumento diseñado y utilizado, y hacia la propuesta formativa llevada a cabo.

De esta manera, se buscó alcanzar una comprensión de la realidad objeto de estudio, desde un posicionamiento singular, descriptivo, heurístico e inductivo (Serrano, 1994) y centrado directamente en la realidad del contexto. Trató de describir y comprender de qué forma el instrumento de autoevaluación tuvo un impacto en el proceso educativo de los árbitros de fútbol y fue un medio útil en acciones formativas participativas, interactivas y colaborativas. Este acercamiento tuvo una orientación hacia un progreso en futuras acciones formativas y una mejora en la práctica. Por ello, se consideró pertinente aplicar este método, adecuado en estudios a pequeña escala, en un espacio y tiempo determinados.

\subsection{Unidades de análisis}

Las unidades de análisis que, concretamente, se estudiaron fueron las siguientes:

- Los informes de autoevaluación realizados por los árbitros. 
- El desempeño en las actuaciones arbitrales en cada dimensión del informe.

- Las necesidades formativas detectadas del grupo en cada una de las jornadas.

- El nivel de satisfacción y la opinión de los árbitros y formadores hacia el instrumento de autoevaluación utilizado.

- La acción formativa desarrollada en el torneo.

\subsection{Participantes}

Una característica que definió a este torneo internacional de fútbol fue la participación de árbitros de distintas nacionalidades que, junto a otro grupo de árbitros adscritos al Comité Guipuzcoano de Árbitros de Fútbol, dirigieron la totalidad de los partidos que se disputaron en el mismo. La acción formativa, que se planteó en la edición del año 2019, tuvo como destinatarios a los árbitros extranjeros inscritos. En ella, intervinieron tres instructores arbitrales pertenecientes al Comité Navarro de Árbitros de Fútbol. En este apartado se presenta el perfil tanto del grupo de árbitros como de los formadores.

Por un lado, en relación a los árbitros que formaron parte de la experiencia, participaron un total de 28 árbitros (Tabla 1) de 9 países distintos: Polonia, Alemania, Países bajos, Turquía, Eslovaquia, Portugal, España, Rumanía y Reino Unido. Como se observa en la tabla, este grupo presentó un perfil heterogéneo. En primer lugar, predominó un perfil de árbitro masculino, con 27 hombres y una mujer. En segundo lugar, la edad media de los participantes fue de 22,2 años. La edad mínima fue de 18 años y la edad máxima de 34 años. En cada país, los árbitros ostentaban una categoría distinta, debido al sistema de competiciones específico de cada territorio. Este aspecto dificulta poder establecer una relación o equivalencia entre ellos. La media de años en la categoría actual era de 2,4 años. El árbitro que más tiempo llevaba en su categoría era de 8 años y el menor de un año. En último lugar, la media del grupo como árbitro en activo era de 5,8 años. El árbitro con mayor tiempo de permanencia en el arbitraje era de 16 años, mientras que el árbitro con menor tiempo en el colectivo era de dos años.

Por otro lado, en la acción formativa intervinieron tres instructores pertenecientes a

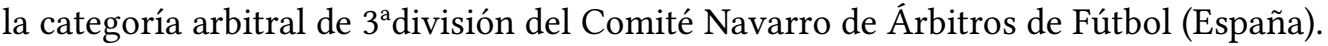
Estas personas eran árbitros en activo, contaban con experiencia en el colectivo arbitral y, además, eran parte del equipo de formación en su respectivo comité donde colaboran en cursos y seminarios de formación permanente. La edad de los instructores era de 32 , 28 y 26 años, y su experiencia en el colectivo era de 18, 14 y 12 años, respectivamente.

\subsection{Instrumentos de obtención de información}

Los instrumentos utilizados para la obtención de información fueron tres: el informe de autoevaluación, el cuestionario de satisfacción y, por último, el grupo de discusión.

En primer lugar, de cara a obtener información sobre el desempeño de los árbitros en sus actuaciones arbitrales durante los partidos y detectar las necesidades del grupo, se utilizó el informe de autoevaluación. El objetivo de este instrumento fue, por un lado, ofrecer a los árbitros participantes un instrumento de autoevaluación para analizar y valorar las distintas actuaciones que llevan a cabo en el torneo. Por otro lado, pretendió servir a los formadores como un medio para recopilar, conocer y organizar la información acerca del desempeño de las actuaciones y detectar las necesidades formativas del grupo en cada una de las jornadas del campeonato.

Las dimensiones y los criterios de evaluación recogidos en el instrumento fueron seleccionados en base a dos documentos: Reglas de Juego 2019-2020 (IFAB, 2019) y la Guía para Delegados de Partido e Informadores de 2 $2^{a}$ División B (CTA, 2018).

La primera versión de la herramienta fue sometida a una evaluación por parte de ocho árbitros de fútbol en activo, con experiencia en el colectivo arbitral y encargados 
Tabla 1. Perfil de los árbitros participantes en la experiencia formativa

\begin{tabular}{|c|c|c|c|c|c|c|}
\hline Árbitro & País & Edad & Género & Categoría & Años categoría & Años arbitraje \\
\hline $\mathrm{A} 1$ & España & 19 & Hombre & Preferente & 1 & 5 \\
\hline A2 & Eslovaquia & 18 & Hombre & Amateur & 3 & 3 \\
\hline A3 & Polonia & 19 & Mujer & 8th Level & 1 & 2 \\
\hline A4 & Rumania & 23 & Hombre & Liga III & 4 & 6 \\
\hline A5 & Rumania & 24 & Hombre & Liga III & 3 & 7 \\
\hline A6 & Rumania & 25 & Hombre & Liga III & 7 & 7 \\
\hline A7 & Polonia & 21 & Hombre & 5th Level & 1 & 3 \\
\hline A8 & Polonia & 23 & Hombre & 3rd Level & 2 & 6 \\
\hline A9 & Turquía & 24 & Hombre & Local Amateur & 3 & 4 \\
\hline A10 & Polonia & 23 & Hombre & 4th Level & 1 & 6 \\
\hline A11 & Rumania & 22 & Hombre & Liga III & 4 & 5 \\
\hline A12 & Rumania & 34 & Hombre & Liga II & 8 & 16 \\
\hline A13 & Portugal & 23 & Hombre & Liga Distrital & 1 & 4 \\
\hline A14 & Rumania & 24 & Hombre & Liga III & 5 & 10 \\
\hline A15 & Rumania & 23 & Hombre & Liga III & 3 & 9 \\
\hline A16 & Rumania & 25 & Hombre & Liga II & 1 & 8 \\
\hline A17 & Países Bajos & 19 & Hombre & KNVB Amateur & 1 & 5 \\
\hline A18 & Alemania & 21 & Hombre & Regional League & 2 & 4 \\
\hline A19 & Reino Unido & 18 & Hombre & 7th Level & 3 & 4 \\
\hline A20 & España & 23 & Hombre & $3^{a}$ División & 1 & 6 \\
\hline A21 & España & 26 & Hombre & $3^{a}$ División & 1 & 10 \\
\hline A22 & España & 21 & Hombre & $1^{\text {a Regional }}$ & 1 & 6 \\
\hline A23 & España & 19 & Hombre & 3ªDivisión & 1 & 6 \\
\hline A24 & España & 22 & Hombre & $1^{\text {a Regional }}$ & 2 & 4 \\
\hline A25 & Portugal & 19 & Hombre & Liga Distrital & 1 & 4 \\
\hline A26 & España & 22 & Hombre & $1^{\mathrm{a}}$ Regional & 3 & 5 \\
\hline A27 & España & 20 & Hombre & $1^{\text {a Regional }}$ & 1 & 3 \\
\hline A28 & España & 21 & Hombre & $1^{\text {a }}$ Regional & 1 & 3 \\
\hline
\end{tabular}

de desarrollar actividades formativas en sus respectivos comités territoriales. Se valoró su adecuación y pertinencia. Es decir, por un lado, si el instrumento contribuye al objetivo para el cual ha sido diseñada, es decir, si los ítems ayudan a recoger información útil para su propuesta formativa, y, por otro lado, si está adaptado a las personas que van a utilizarla. Recogidas las valoraciones de los expertos, se analizaron y consideraron todas las sugerencias y aspectos de mejora para la elaboración final del instrumento. Finalmente, se decidió presentar el recurso en formato digital (Google Forms). En el apartado posterior se describe tanto su estructura general y su proceso de elaboración.

En segundo lugar, para determinar la experiencia de los árbitros participantes al usar el instrumento de evaluación como recurso para analizar y valorar sus actuaciones en los partidos, y hacia la experiencia formativa en su conjunto, se administró un cuestionario a los 28 árbitros participantes al finalizar la actividad formativa. La encuesta se realizó a través de un formulario en línea, de carácter anónimo, que la totalidad de los participantes cumplimentó y remitió voluntariamente. El cuestionario constó de doce preguntas de respuesta cerrada (Tabla 2), con formato de escala Likert, y una pregunta 
de respuesta abierta para reflejar otro tipo de cuestiones tanto sobre el instrumento de autoevaluación como de la experiencia educativa.

Tabla 2. Aspectos evaluados por los participantes en el cuestionario
1. Utilizar distintos dispositivos móviles para realizar el informe es positivo
2. El instrumento de autoevaluación utilizado tiene un manejo sencillo
3. La herramienta utilizada tiene un formato, una estructura y una organización adecuada
4. El tiempo dedicado para elaborar el informe de autoevaluación es adecuado
5. La herramienta permite un análisis y una valoración global sobre la actuación
6. Los aspectos incluidos en el instrumento son adecuados para una evaluación completa
7. La rúbrica, sus descriptores y las orientaciones ayudan a realizar el informe
8. Señalar los aspectos positivos y con recorrido de mejora es útil y relevante
9. Los encuentros de formación generan la participación e interacción de los participantes
10. El feedback recibido en las sesiones es útil y contribuye a la mejora y al progreso
11. Los aspectos con recorrido de mejora y las temáticas seleccionadas son acertadas
12. El uso de vídeos como material didáctico es un recurso útil

El cuestionario fue validado mediante la técnica de juicio de expertos, cuya realización adecuada desde un punto de vista metodológico constituye a veces el único indicador de validez de contenido del instrumento de recogida de datos o de información (Escobar-Pérez y Cuervo-Martínez, 2008). Tratándose de un instrumento de elaboración propia, fue validado mediante un procedimiento metodológicamente adecuado, por lo que se sometió a validación por juicio de expertos. Las respuestas y sugerencias fueron procesadas, analizadas y consideradas para la construcción de la versión final de un cuestionario útil para su propósito y con validez científica, es decir, el grado en que un instrumento de medida mide aquello que realmente pretende medir o sirve para el propósito para el que ha sido construido (Arribas, 2004).

Por último, y en tercer lugar, para conocer la opinión de los formadores sobre la utilidad y las aportaciones del instrumento de autoevaluación como fuente de información para conocer el desempeño y detectar las necesidades de los árbitros en el torneo, se llevó a cabo un grupo de discusión con los tres instructores que desarrollaron la experiencia de formación para contrastar pareceres con relación a la utilidad del instrumento diseñado para elaborar los informes de autoevaluación, la información recibida sobre el desempeño de las actuaciones arbitrales, su transferencia a las sesiones de formación, así como la valoración general de la experiencia, en cuanto a la metodología utilizada, los recursos empleados, las dinámicas de grupo generadas, etc.

\subsection{Trabajo de campo}

La práctica educativa se desarrolló en el Torneo de Fútbol Internacional Donosti Cup, concretamente, en la edición del año 2019. Este campeonato se celebra anualmente en la ciudad de San Sebastián (España) durante el mes de julio. En el evento, se dan cita jugadores, oficiales, árbitros y aficiones de distintas zonas geográficas, tanto a nivel nacional como internacional. La primera edición tuvo lugar el 1992 y participaron 32 equipos de tres países diferentes: España, Francia e Italia. Actualmente, más de 80.000 jugadores de 69 países forman parte de este campeonato que, a lo largo de los años, se ha convertido en uno de los torneos más grandes a nivel mundial.

En la Figura 1, se presenta globalmente el enfoque de la propuesta de formación que tuvo lugar con los árbitros en el torneo. Comprendió las siguientes fases: designación de partidos de fútbol en cada jornada, elaboración de los informes de autoevaluación a través del instrumento diseñado para este fin, detección de las necesidades y de los aspectos con recorrido de mejora del grupo, y desarrollo de los encuentros formativos. 

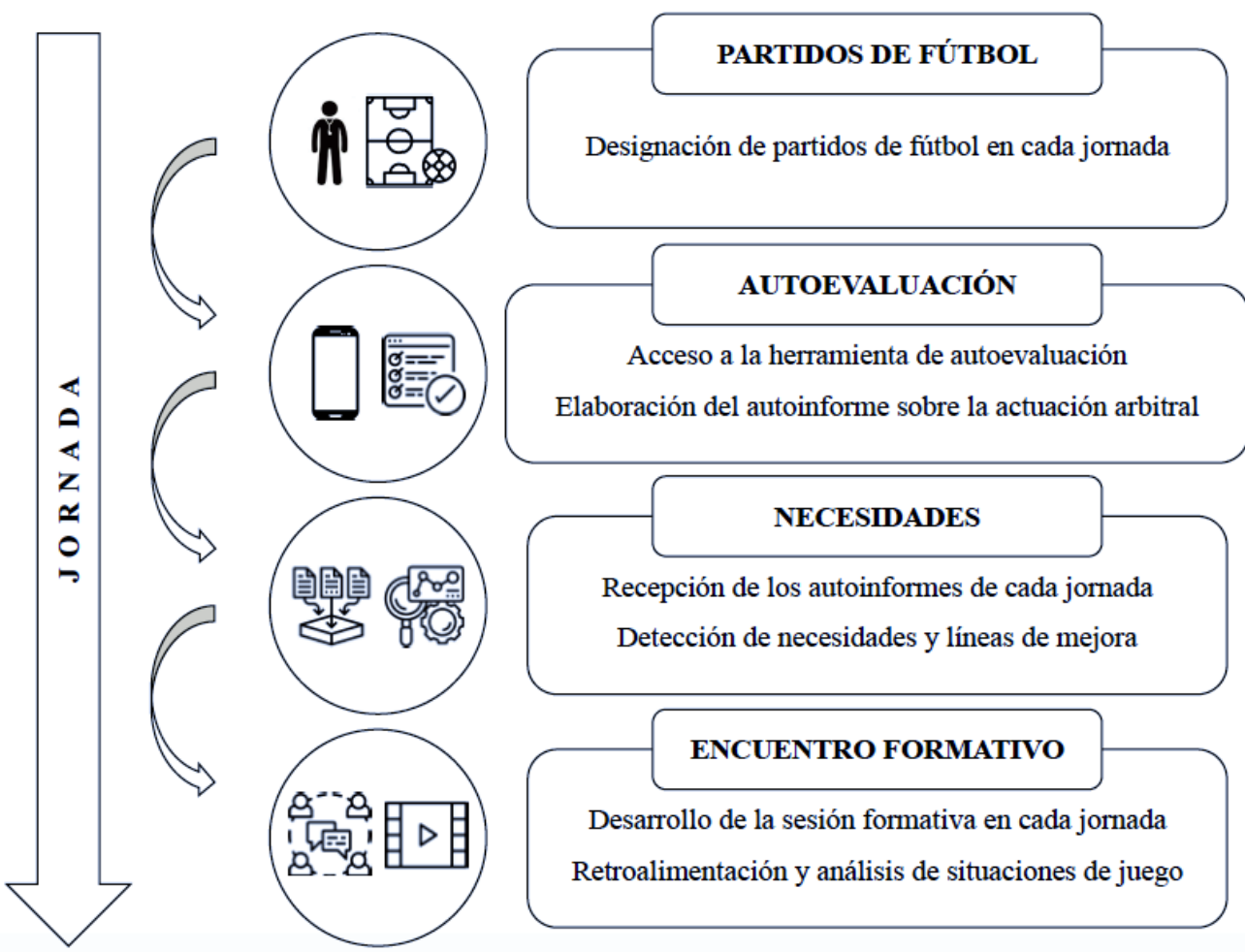

Figura 1. Enfoque pedagógico de la experiencia formativa

Durante las jornadas del torneo, se disputaron una gran cantidad de partidos de fútbol. Contó con categorías masculinas y femeninas, en modalidad de fútbol $11 \mathrm{y}$ fútbol 8. Las edades de los jugadores se encontraban entre los 10 y los 18 años. El formato de la competición constó de una primera fase de grupos y por una fase final de eliminatorias. Los partidos de fútbol se disputaron en terrenos de juego situados en San Sebastián y en localidades próximas. En esta edición, el grupo de árbitros dirigió un total de 371 partidos. Este número corresponde con la totalidad de los informes de autoevaluación que realizaron los participantes en cada una de las jornadas del torneo (Tabla 3).

Tabla 3. Informes de autoevaluación realizados en cada jornada

\begin{tabular}{ll}
\hline Jornada & Informes de autoevaluación \\
\hline Jornada 1 & 74 \\
Jornada 2 & 75 \\
Jornada 3 & 72 \\
Jornada 4 & 98 \\
Jornada 5 & 52 \\
Total & 371 \\
\hline
\end{tabular}

En cada jornada, los árbitros recibieron previamente las designaciones con el número específico de partidos que debían dirigir en cada una de ellas. Así, tras cada encuentro, elaboraron los informes de autoevaluación. A continuación, se describe la estructura general y el proceso de elaboración del informe de autoevaluación (Figura 2).

Inicialmente, en cada uno de los partidos, el usuario registró los siguientes datos: nombre del árbitro, país, jornada del campeonato y categoría del partido. Esta informa- 


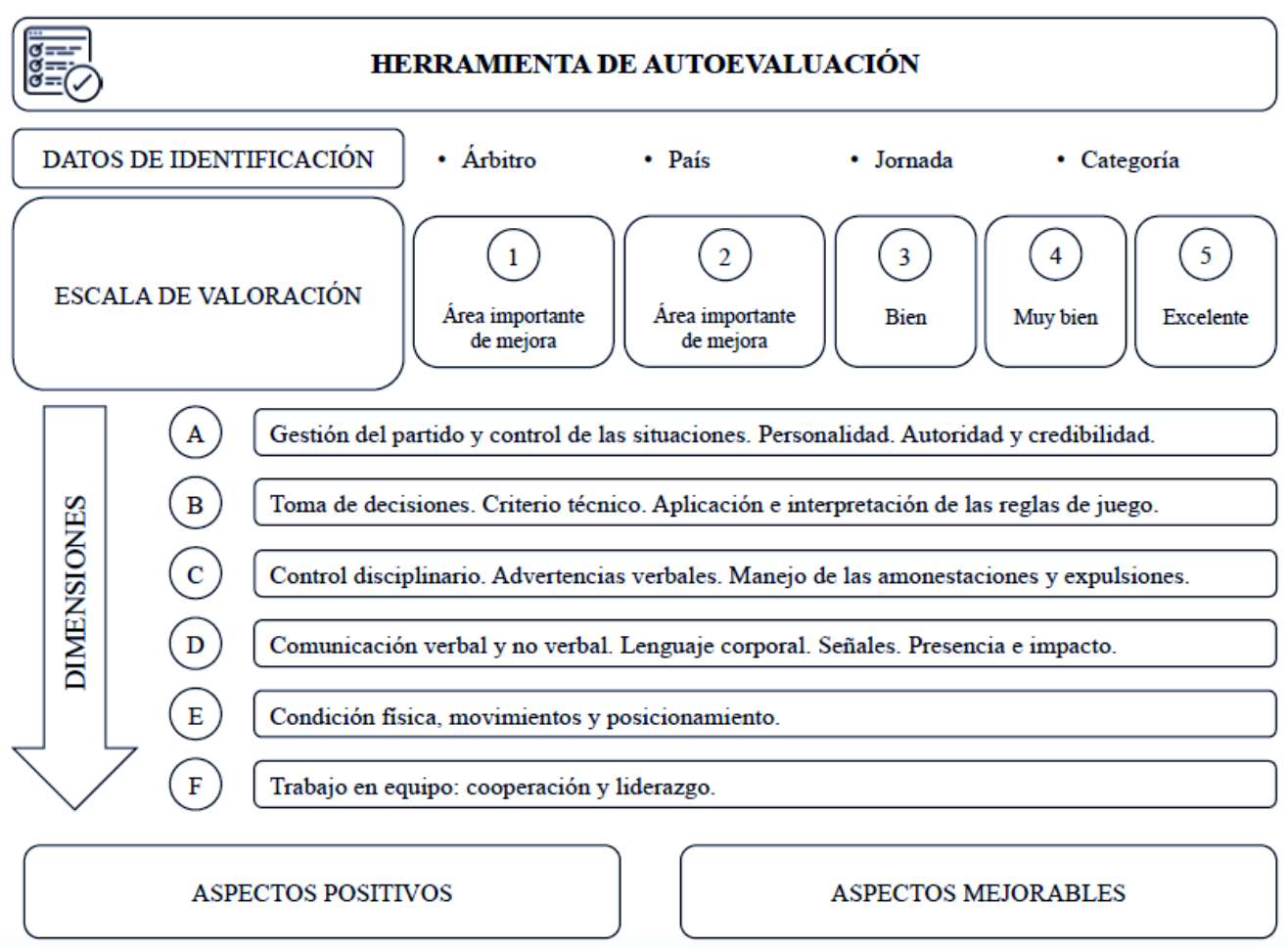

Figura 2. Estructura del instrumento de autoevaluación

ción permitió recopilar y organizar los autoinformes para llevar a cabo un seguimiento adecuado del desempeño de las actuaciones durante el torneo.

Posteriormente, la autoevaluación de cada actuación giró en torno a seis apartados: (a) Gestión del partido y control de las situaciones. Personalidad. Autoridad y credibilidad; (b) Toma de decisiones. Criterio técnico. Aplicación e interpretación de las reglas de juego; (c) Control disciplinario. Advertencias verbales. Manejo de las amonestaciones y expulsiones; (d) Comunicación verbal y no verbal. Lenguaje corporal. Señales. Presencia e impacto; (e) Condición física, movimientos y posicionamiento; (f) Trabajo en equipo: cooperación y liderazgo.

Cada una de las dimensiones se evaluó atendiendo a la siguiente escala de valoración: (1) Importante área de mejora; (2) Pequeña área de mejora; (3) Bien; (4) Muy bien; (5) Excelente. Esta escala se complementó con una rúbrica de evaluación, que ofreció las orientaciones necesarias para que los árbitros realizasen su informe tras cada partido. Este recurso aportó los descriptores correspondientes a cada nivel de ejecución en cada una de las dimensiones. Debido a su extensión, y a modo de ejemplo, se presenta el apartado relacionado con el control disciplinario (Tabla 4).

En este ejemplo, se observa la escala de valores para evaluar esta dimensión concreta. A cada nivel de ejecución se le asignó un descriptor con información detallada y los aspectos principales a valorar. Este recurso complementario pretendió consensuar qué aspectos son abordados y su nivel de desempeño, garantizar la comprensión del instrumento y encontrar un criterio uniforme en la elaboración del autoanálisis.

Para finalizar su proceso de elaboración, se seleccionaron hasta un máximo de tres aspectos positivos y tres con recorrido de mejora, de acuerdo a un listado posible de 20 ítems, recogidos según distintas temáticas relacionadas con el arbitraje (por ejemplo, entradas y disputas, ocasión manifiesta de gol, control del partido, confrontación de jugadores, trabajo en equipo, lenguaje corporal, etc.). Esta información, junto a la valoración de cada una de las dimensiones, permitió detectar los aspectos mejorables y las necesidades, tanto a nivel individual como grupal. 
Tabla 4. Rúbrica de evaluación sobre el control disciplinario

Apartado 3. Control disciplinario.

1 Partido muy exigente con muchas situaciones difíciles. No tiene consistencia y buen criterio disciplinario en las situaciones de juego cruciales (temeridad, juego brusco grave, conducta violenta, ocasión manifiesta de gol, ataque prometedor, etc.).

2 Partido exigente con algunas situaciones difíciles. No tiene consistencia y buen criterio disciplinario en las situaciones de juego cruciales (temeridad, juego brusco grave, conducta violenta, ocasión manifiesta de gol, ataque prometedor, etc.).

3 Partido con poca exigencia y sin situaciones difíciles, dentro de los cauces deportivos. Toma decisiones disciplinarias de forma correcta en las situaciones que lo requieren.

4 Partido exigente con algunas situaciones difíciles. Tiene consistencia y buen criterio disciplinario en las situaciones de juego cruciales (temeridad, juego brusco grave, conducta violenta, ocasión manifiesta de gol, ataque prometedor, etc.).

5 Partido muy exigente con muchas situaciones difíciles. Tiene consistencia y buen criterio disciplinario en las situaciones de juego cruciales (temeridad, juego brusco grave, conducta violenta, ocasión manifiesta de gol, ataque prometedor, etc.).

Tras finalizar cada jornada, el equipo docente se reunió con el grupo de árbitros participantes para desarrollar los encuentros formativos. Previamente, los formadores revisaron la información recibida en las valoraciones sobre las actuaciones arbitrales durante la jornada para detectar las necesidades del grupo y los distintos aspectos a mejorar. Esta información tuvo una vinculación estrecha con las sesiones formativas.

Dependiendo de las áreas de mejora observadas en las valoraciones, se establecieron las temáticas para desarrollar en cada sesión, usando el vídeo como principal recurso didáctico. Se utilizó el material audiovisual Refereeing Assistance Programme (RAP) 2019, elaborado por la Union of European Football Associations (UEFA) (UEFA, 2019). Este recurso ofrece un amplio repositorio de vídeos sobre situaciones de juego de distintas competiciones, clasificados en distintas temáticas (Figura 3).

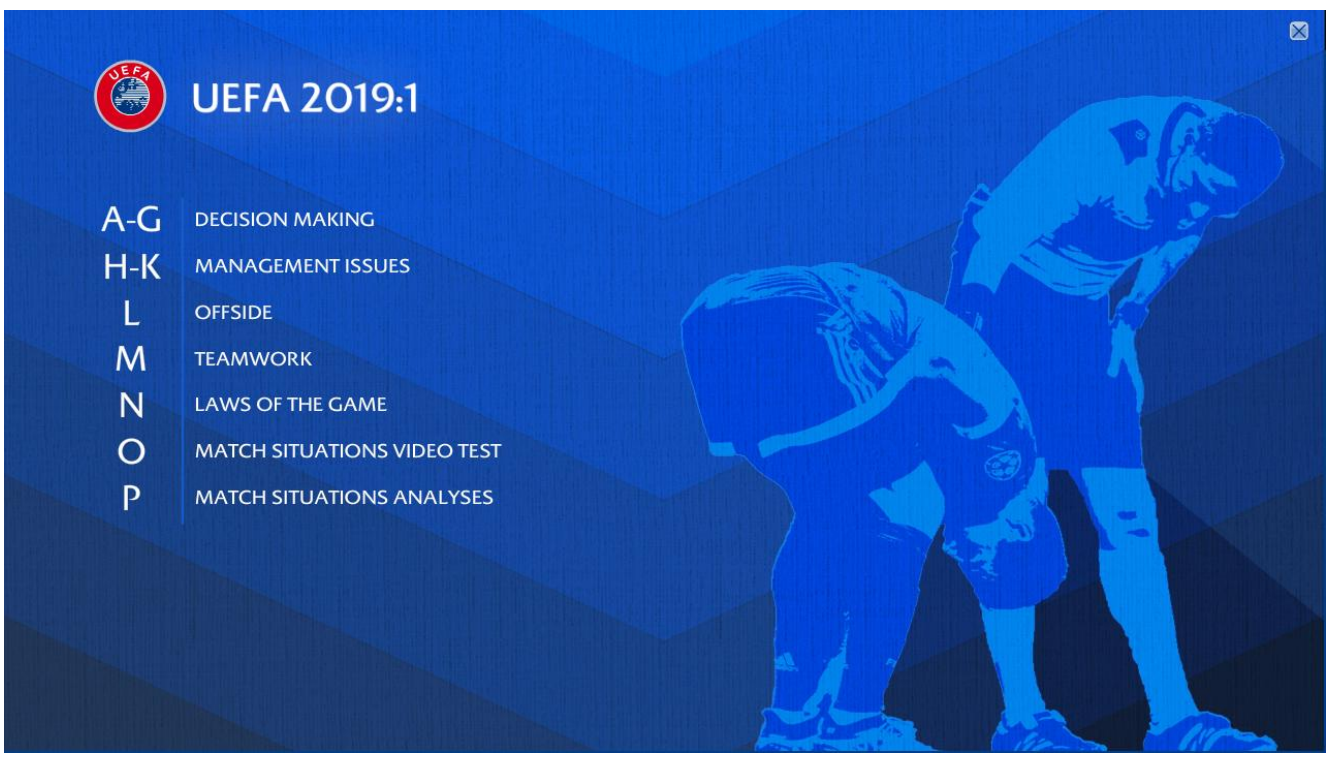

Figura 3. Indice de contenidos del Refereeing Assistance Programme (RAP) 2019

En cuanto a la dinámica de trabajo, se generó un ambiente de aprendizaje social, dinámico e interactivo, donde el formador orientó los debates y discusiones. En primer lugar, se introdujo la temática elegida y su justificación, presentando los aspectos con recorrido de mejora detectados. Los participantes tuvieron la oportunidad de compartir con el grupo la descripción y decisión de situaciones ocurridas en sus partidos de 
la jornada. En segundo lugar, el docente proporcionó unas consideraciones estándar para el análisis de jugadas y la toma de decisiones, según la temática. En tercer lugar, se analizaron distintas situaciones de juego, teniendo en cuenta las directrices, generando debate y discusión en el grupo. Se identificaron los elementos clave de cada clip de vídeo, argumentando y determinando la decisión técnica y disciplinaria, y razonando la decisión. También se aportaron distintas herramientas para el control y gestión del partido. Por último, se realizó una puesta en común con las conclusiones de la sesión.

\subsection{Tratamiento de la información}

El informe de autoevaluación se envió a los árbitros participantes a través de un enlace a Google Forms, en la sesión de bienvenida y antes de la primera jornada del torneo. Esta herramienta de administración de formularios permitió la recepción continua de los autoinformes. Estos datos se volcaron en una hoja de cálculo Microsoft Excel. Al finalizar cada jornada, se analizaron las valoraciones de los árbitros en cada una de las dimensiones del informe, los aspectos positivos y mejorables, con el fin de detectar las necesidades y las líneas de mejora del grupo. Los datos se procesaron, considerándose la media y la mediana como medidas de tendencia central, proporcionando un número que resumiese la distribución media de los valores. El máximo, mínimo y la desviación estándar se tomaron como medidas de dispersión respecto a la medida de la tendencia central. La información fue clasificada utilizando el servicio de alojamiento de archivos en la nube Google Drive para facilitar un acceso inmediato a los datos por los docentes.

El cuestionario de satisfacción se administró a los árbitros participantes a través de un enlace a Google Forms, en la sesión final de la formación. El análisis estadístico de los resultados del cuestionario se realizó con el paquete IMB SPSS Statistics V25. Se calculó la frecuencia y porcentaje de las respuestas a cada pregunta cerrada. Las respuestas a la pregunta abierta del cuestionario se procesaron mediante el programa Atlas.ti V8., permitiendo establecer un conjunto de aspectos recogidos en categorías.

El grupo de discusión con los tres instructores se realizó al finalizar la acción formativa. El objetivo era conocer su opinión sobre la utilidad y las aportaciones del instrumento de autoevaluación como fuente de información, así como la metodología utilizada en las sesiones de trabajo, los recursos empleados, la dinámica desarrollada, etc. La dinámica generada fue grabada y posteriormente transcrita, analizando su contenido mediante el programa Atlas.ti V8. El sistema de codificación utilizado fue descriptivo, inductivo y abierto. Se clasificaron en categorías los temas que se repitieron en sus discursos y que estuvieron relacionados con los distintos aspectos didácticos de la experiencia educativa desarrollada.

\section{RESULTADOS}

\subsection{Informes de autoevaluación y desempeño arbitral}

La Tabla 5 muestra la puntuación media obtenida en cada una de las dimensiones que componen el instrumento de autoevaluación. Se consideraron todos los informes de autoevaluación realizados en los partidos de cada una de las jornadas. Los datos ofrecen una visión global del desempeño de las actuaciones arbitrales en cada apartado:

- La primera dimensión, vinculada fundamentalmente con el control y la gestión del partido, obtuvo una puntuación baja en la primera jornada $(2,54)$ y en la quinta jornada $(2,31)$. Durante el resto de jornadas mantuvo una valoración similar, aunque fue ligeramente inferior al nivel deseado.

- La segunda dimensión, relacionada principalmente con el criterio técnico en la toma de decisiones, tuvo una puntuación satisfactoria en la primera jornada $(3,22)$. Sin embargo, obtuvo una valoración muy baja en la segunda $(2,15)$, tercera $(2,46)$ y 
cuarta jornada $(2,09)$.

- La tercera dimensión, emparejada con el control disciplinario y el uso de las advertencias verbales, presentó unas puntuaciones regulares durante las jornadas, pero se encontraron en un nivel inferior a un desempeño adecuado. La cuarta dimensión, relacionada con la comunicación verbal y no verbal, tuvo una puntuación aceptable y regular durante la primera, segunda y tercera jornada. Sin embargo, presentó una baja puntuación en la quinta jornada $(2,40)$.

- La quinta dimensión, vinculada con la condición física y el posicionamiento, obtuvo unas puntuaciones positivas, por encima del nivel esperado.

- La sexta dimensión, relacionada con el trabajo en equipo, también presentó unas puntuaciones superiores y al nivel deseado en cada una de las jornadas.

Tabla 5. Puntuación media de las dimensiones del informe de autoevaluación en cada jornada

\begin{tabular}{|c|c|c|c|c|c|}
\hline \multicolumn{6}{|l|}{ Dimensión del informe } \\
\hline $\begin{array}{l}\text { Apartado } 1 . \text { Gestión del partido. Personalidad. } \\
\text { Autoridad y credibilidad. Manejo de las } \\
\text { confrontaciones. Observaciones y protestas. }\end{array}$ & 2,54 & 2,81 & 2,81 & 2,86 & 2,31 \\
\hline $\begin{array}{l}\text { Apartado 2. Toma de decisiones. Criterio } \\
\text { técnico. Aplicación e interpretación de las reglas } \\
\text { de juego. Evaluación de situaciones específicas. }\end{array}$ & 3,22 & 2,15 & 2,46 & 2,09 & 2,71 \\
\hline $\begin{array}{l}\text { Apartado 3. Control disciplinario. Advertencias } \\
\text { verbales. Manejo de amonestaciones y } \\
\text { expulsiones. }\end{array}$ & 2,81 & 2,81 & 2,75 & 2,76 & 2,75 \\
\hline $\begin{array}{l}\text { Apartado 4. Comunicación verbal y no verbal. } \\
\text { Presencia e impacto. }\end{array}$ & 2,73 & 3,00 & 3,00 & 3,00 & 2,40 \\
\hline $\begin{array}{l}\text { Apartado } 5 \text {. Condición física, movimientos y } \\
\text { posicionamiento. }\end{array}$ & 2,97 & 3,29 & 3,19 & 3,21 & 3,44 \\
\hline $\begin{array}{l}\text { Apartado } 6 . \text { Trabajo en equipo. Cooperación y } \\
\text { liderazgo. }\end{array}$ & 3,46 & 3,24 & 3,32 & 3,21 & 3,33 \\
\hline
\end{tabular}

\subsection{Detección de necesidades formativas en el grupo}

La Tabla 6 recoge la frecuencia de los aspectos con recorrido de mejora señalados por los árbitros en sus informes de autoevaluación durante cada jornada. Esta información permitió al equipo docente detectar las necesidades específicas del grupo:

- En la primera jornada, se observaron un total de ocho puntos con recorrido de mejora. Destacaron, con mayor frecuencia, el control y la lectura del partido, la confrontación de jugadores y, por último, las observaciones y protestas.

- En la segunda jornada, se encontraron un total de siete aspectos de mejora. Sobresalieron las acciones de mano, de sujetar y la aplicación de la ventaja.

- En la tercera jornada, se recogieron cuatro puntos mejorables. Las simulaciones y los incidentes en el área de penal presentaron mayor frecuencia.

- En la cuarta jornada, se observaron ocho aspectos con recorrido de mejora. Despuntan las entradas y disputas, junto al uso ilegal de los brazos.

- En la quinta jornada, presentó siete puntos mejorables. El control de los jugadores, el control de los oficiales y el lenguaje corporal destacaron. 
Tabla 6. Necesidades formativas detectadas en las distintas jornadas

\begin{tabular}{llllll}
\hline \multirow{2}{*}{ Aspectos } & \multicolumn{5}{c}{ Frecuencia } \\
& J1 & J2 & J3 & J4 & J5 \\
\hline Control y lectura del partido & 20 & - & - & - & - \\
Confrontación de jugadores & 20 & - & - & - & - \\
Observaciones y protestas & 20 & - & - & - & - \\
Simulaciones & 12 & - & 16 & - & - \\
Posicionamiento & 12 & - & - & 8 & - \\
Ataque prometedor & 7 & 9 & 10 & - & - \\
Acciones de mano & 7 & 25 & - & 5 & 3 \\
Acciones de sujetar & 6 & 25 & - & 4 & 2 \\
Aplicación de la ventaja & - & 25 & - & - & - \\
Ocasión manifiesta de gol & - & 9 & 10 & - & - \\
Gestión de los tiros libres & - & 5 & - & 12 & - \\
Control de las reanudaciones & - & 5 & - & 12 & - \\
Incidentes en el área de penal & - & - & 17 & - & - \\
Entradas y disputas & - & - & - & 24 & 8 \\
Uso ilegal de los brazos & - & - & - & 24 & 5 \\
Fuera de juego & - & - & - & 8 & - \\
Control de los jugadores & - & - & - & - & 20 \\
Control de los oficiales & - & - & - & - & 20 \\
Lenguaje corporal & - & - & - & - & 20 \\
\hline
\end{tabular}

\subsubsection{Nivel de satisfacción y opinión de los árbitros sobre el instrumento de autoevaluación y la experiencia formativa}

En general, los resultados de la experiencia educativa fueron considerados como muy satisfactorios (Figura 4). A continuación, se detallan los resultados obtenidos en cada una de las cuestiones, así como las aportaciones y consideraciones realizadas:

- El $100 \%$ de los árbitros se mostraron muy de acuerdo al considerar la utilidad e interés en usar los propios dispositivos móviles para realizar los informes de autoevaluación. "Es cómodo hacer los informes desde nuestro teléfono móvil" (A3). "El enlace y código QR facilita el acceso a la herramienta" (A14). "Desde nuestro teléfono puedes hacer el informe con más rapidez" (A9).

- El $64 \%$ de los participantes se muestra muy de acuerdo y el $36 \%$ de acuerdo al valorar la sencillez en el manejo de la herramienta utilizada. "El instrumento es fácil de utilizar" (A7). "Realizar la valoración es sencillo" (A12).

- Un $79 \%$ se mostró muy de acuerdo y un $21 \%$ de acuerdo sobre la adecuación en el formato, estructura y organización del instrumento utilizado. "Tiene un formato sencillo y una buena presentación" (A15). "La organización es adecuada porque puedes valorar cada apartado paso a paso" (A28).

- En relación al tiempo empleado en la realización del informe, hubo mayores diferencias en las valoraciones. El 18\% de los participantes se mostraron en desacuerdo, mientras que el resto se mostró muy de acuerdo o de acuerdo. "Cuando tienes partidos seguidos, no tienes tiempo suficiente para hacer el informe con tranquilidad" (A25). "Necesitas tiempo después de cada partido para reflexionar y analizar tu actuación" (A4). "Si tuviésemos un descanso entre cada uno de los partidos, 
podríamos analizar mejor el partido" (A9).

- El 57\% de los participantes se mostró muy de acuerdo y el $43 \%$ de acuerdo al considerar si la herramienta facilita el análisis, valoración y reflexión de las actuaciones arbitrales en un partido. "Es muy interesante porque te ayuda a reflexionar sobre cómo has actuado" (A16). "Me parece importante tener recursos para analizar cómo dirigidos los partidos" (A3). "Te ayuda a pensar y valorar la actuación sobre los apartados importantes" (A13). "Es una ayuda para pararte y pensar en los aspectos que puedes mejorar" (A1).

- El 92\% se mostró muy de acuerdo o de acuerdo al valorar si las dimensiones que se incluyen en el instrumento son adecuadas para realizar una evaluación completa de la actuación. Sin embargo, el 8\% se mostró en desacuerdo. "Se puede hacer una valoración más amplia, pero trata aspectos importantes" (A5). "Los bloques que evaluamos son los más importantes en la actuación de un árbitro" (A26). "Te permite una valoración global y por apartados" (A7).

- El $61 \%$ de los participantes se mostró muy de acuerdo y el $39 \%$ de acuerdo al considerar si la rúbrica de evaluación y las orientaciones facilitadas ayudan a la elaboración del informe. "La rúbrica ayuda para saber valorar los bloques con unos criterios claros" (A18). "Es importante para saber qué puntuación es la más adecuada en cada apartado" (A21). "Las orientaciones te ayudan analizar mejor la actuación según las decisiones que has tomado" (A26).

- El 57\% de los árbitros se mostraron muy de acuerdo y el $43 \%$ de acuerdo en cuanto al interés en tener la oportunidad de señalas los aspectos destacables y mejorables tras una actuación. "A veces es difícil pensar en los aspectos que necesitamos mejorar" (A6). "Nos hace pensar sobre cada aspecto y aquellos puntos en los que podemos mejorar" (A7). "Tienes crítico y sincero para ver los puntos mejorables" (A28). "Ser consciente de los puntos a mejorar es muy importante y también tener ayuda sobre cómo hacerlo" (A14). "Es como una especie de cuaderno de bitácora donde marcas tus aprendizajes" (A15).

- El $71 \%$ se mostró muy de acuerdo y el $29 \%$ de acuerdo al considerar si los encuentros de formación tuvieron un enfoque participativo e interactivo entre los formadores y los participantes. "Nos ha permitido dialogar y discutir con los compañeros y con los profesores" (A2). "Es interesante debatir sobre las distintas situaciones que nos ocurren en los partidos, aprendemos todos" (A9). "Participar y debatir es importante porque todos aprendemos de todos" (A7). "Puedes ver otros puntos de vista, rebatir y llegar a conclusiones" (A19).

- El 93\% de los participantes se mostró muy de acuerdo o de acuerdo, mientras que el 7\% se mostró en desacuerdo, al valorar la utilidad del feedback recibido por los docentes en las sesiones formativas. "Los comentarios analizados por los formadores han sido útiles y muy prácticos" (A4). "El profesor analiza los informes y se centra en los aspectos que el grupo necesita mejorar" (A20). "La retroalimentación y las orientaciones prácticas nos ayudan a mejorar en las siguientes actuaciones en el campo" (A13). "Se nota que los aspectos que marcamos como mejorables se trabajan con recursos interesantes" (A17). "El feedback está relacionado con los puntos a mejorar que se ven" (A19).

- El 68\% de los participantes se mostró muy de acuerdo, el 25\% de acuerdo y el $7 \%$ se mostró en desacuerdo sobre el acierto al detectar los aspectos mejorables y las temáticas seleccionadas para trabajar en las sesiones. "Los temas están bien planteados y ayudan a la mejora del grupo" (A6). "Las temáticas que se han trabajado en cada clase están relacionados con los puntos a mejorar que se encontraban en cada jornada" (A3). "Cada día se han trabajado temas específicos y han sido unas 
clases muy prácticas" (A18). "Hemos podido trabajar contenidos relacionados con las reglas y los temas más importantes" (A17). "Una buena manera para ver los errores y progresar" (A24).

- El 89\% de los participantes se mostraron muy de acuerdo y el $11 \%$ de acuerdo cuando valoran la utilidad del vídeo como material didáctico y su uso en los encuentros de formación. "Los vídeos son muy interesantes porque ves distintas situaciones que tienes que juzgar con unos criterios claros" (A5). "Trabajar en las sesiones con vídeos es muy útil porque trabajar los conceptos importantes para aplicar en el campo" (A8). "Se pueden trabajar y analizar los aspectos importantes y la manera de aplicarlos" (A11). "Permite sacar buenas conclusiones y directrices prácticas" (A9). "Existe una gran variedad de temáticas" (A23). "Usar los vídeos nos ha permitido analizar las jugadas en grupo, participar, dialogar y llegar a conclusiones" (A19).

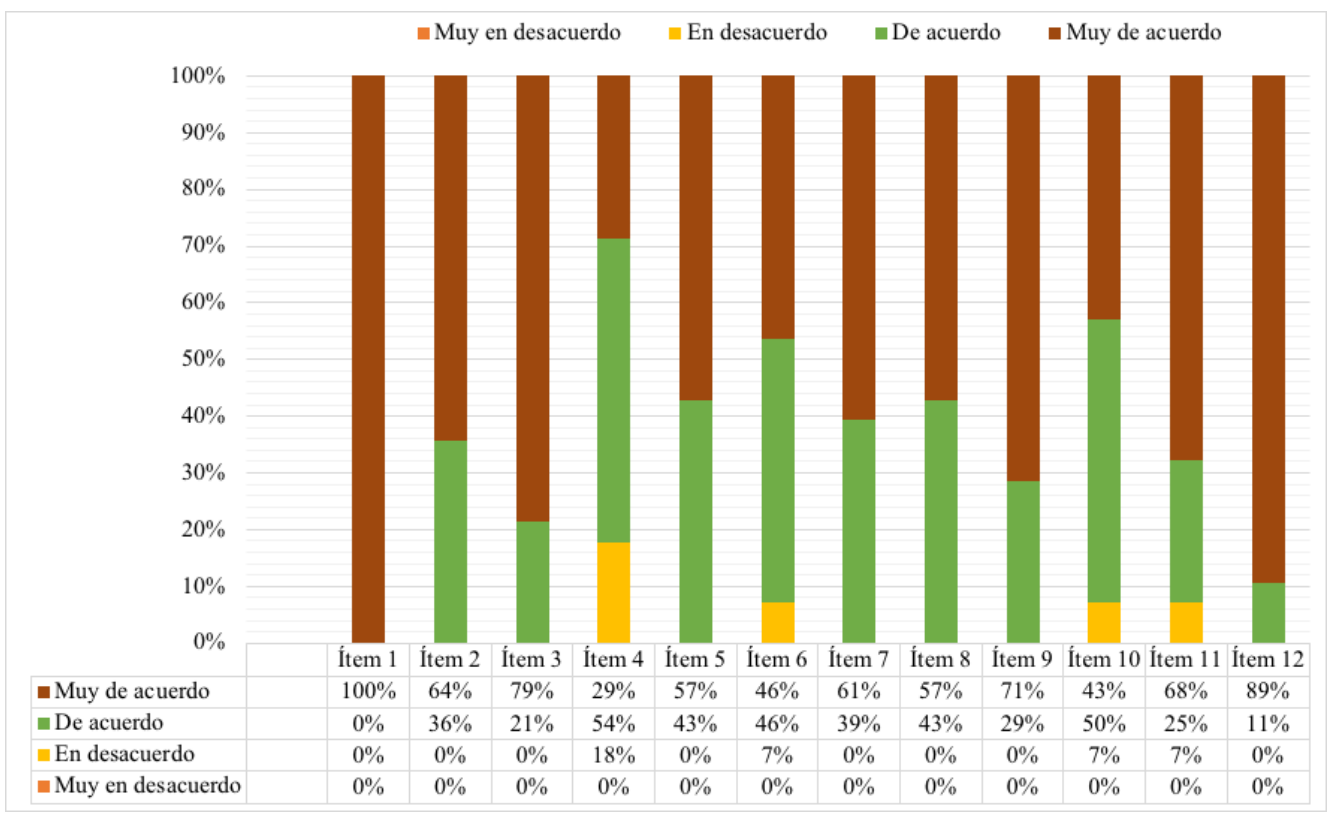

Figura 4. Nivel de satisfacción de los participantes hacia la experiencia formativa

\subsection{Nivel de satisfacción y opinión de los instructores sobre el instrumento de autoevaluación y la acción formativa}

Al finalizar la experiencia formativa, se llevó a cabo un grupo de discusión, en el que participaron los tres profesores que desarrollaron la formación. Se les solicitó su opinión sobre la utilidad del instrumento diseñado para elaborar los informes de autoevaluación, sobre la información recibida a través de los mismos durante cada jornada y sobre el desarrollo de los encuentros de formación. Sus aportaciones se analizaron, codificándolas y categorizándolas para ofrecer una serie de conclusiones:

- La coherencia entre el diseño de la experiencia y su desarrollo efectivo, pese a tratarse del primer ensayo de implantación en este torneo con sus árbitros.

- La utilidad del instrumento diseñado para que los árbitros realicen los informes de autoevaluación. Los participantes han acogido muy bien esta herramienta y han entendido que contribuye a su formación y a la mejora de sus actuaciones.

- De cara a mejorar el instrumento, cabe la posibilidad de ampliar sus apartados y, en cada uno de ellos, desglosarlos en otros ítems que sean más específicos y estén 
estrechamente relacionados con cada una de las dimensiones propuestas.

- Para el equipo docente, la herramienta ha sido valiosa ya que les ha permitido recibir información del desempeño diario de los usuarios, en torno a una serie de dimensiones fijadas en el instrumento y detectar puntos de mejora.

- La información recabada en cada jornada ha facilitado extraer un conjunto de necesidades y ha ayudado a seleccionar las temáticas y los recursos didácticos para trabajar en cada una de las sesiones de formación junto a los árbitros.

- La importancia de los procesos de feedback en las sesiones de formación. Este aspecto cobra importancia dentro de un enfoque centrado en el diálogo entre los participantes y en su proceso de evaluación continua. La retroalimentación es un elemento esencial que favorece un espacio compartido de trabajo donde todos los miembros de la comunidad aprenden y se enriquecen en grupo.

- La percepción del logro generalizado de un clima de aprendizaje muy positivo, caracterizado por el interés de los árbitros, su participación, su colaboración y la interacción generada dentro del grupo. El aprovechamiento ha sido positivo.

- El elevado nivel de adquisición de contenidos específicos sobre las reglas de juego y sobre las estrategias relacionadas con el control y gestión del partido. Los usuarios manifiestan su utilidad y contribución a la mejora en transferir estos aprendizajes al terreno de juego. Destacan la importancia de la formación continua, del uso del vídeo como recurso educativo y del enfoque utilizado.

- La importancia de los procesos de feedback en las sesiones de formación. Este aspecto cobra importancia dentro de un enfoque centrado en el diálogo entre los participantes y en su proceso de evaluación continua. La retroalimentación es un elemento esencial que favorece un espacio compartido de trabajo donde todos los miembros de la comunidad aprenden y se enriquecen en grupo.

\section{DISCUSIÓN Y CONCLUSIONES}

Este artículo presenta el escenario educativo que surge con el desarrollo de una acción formativa dirigida a los árbitros participantes en un torneo internacional de fútbol. La práctica incorpora el informe de autoevaluación como una pieza clave del proceso formativo. Por un lado, ofrece a los usuarios un instrumento de autoevaluación para analizar y valorar su actividad en los partidos dirigidos en el campeonato. Por otro lado, facilita a los formadores una fuente de información para conocer el desempeño de las actuaciones de los árbitros y detectar las necesidades del grupo en cada jornada. Esta información, previamente analizada, se traslada a las sesiones de formación donde los instructores facilitan procesos de feedback dialógico, seleccionan las temáticas a tratar y establecen vinculación con los materiales didácticos específicos, todo ello desde un enfoque metodológico basado en la participación, la interacción y la colaboración.

Los resultados obtenidos evidencian un alto grado de satisfacción y aceptación hacia el instrumento de autoevaluación, los recursos y materiales interactivos (Armenteros, Liaw, et al., 2013; Armenteros et al., 2017), los espacios y tiempos de formación, la calidad de la retroalimentación recibida (Nicol, 2011) y, en último lugar, hacia las estrategias metodológicas empleadas en las sesiones de trabajo para promover la participación, la interacción y la colaboración (Armenteros et al., 2019; Recio-Moreno et al., 2020). El informe de autoevaluación tiene un impacto positivo en los procesos de formación y mejora continua de los árbitros, considerándose un medio útil para el análisis y la reflexión sobre la actividad deportiva. Se valora positivamente el compromiso, la responsabilidad y la actitud receptiva que los usuarios mantienen hacia el 
uso del instrumento y hacia la comprensión del feedback que fundamenta la propuesta formativa (Nicol, 2011; Price et al., 2010).

La información extraída de los informes de autoevaluación permite conocer el desempeño de los árbitros, tanto a nivel individual como grupal, en sus actuaciones arbitrales y detectar las áreas de mejora dentro del grupo. Las valoraciones otorgadas en cada una de las dimensiones del instrumento, los aspectos positivos y los aspectos mejorables favorecen una visión global del rendimiento y específico en cuanto a cada una de las parcelas de valoración. De este modo, la información se considera útil de cara a adaptar y personalizar el contenido abordado en las sesiones de trabajo puesto que ahonda en las líneas de mejora que se contemplan en los autoanálisis. Ayuda a abordar conocimientos específicos sobre las reglas de juego, analizar de situaciones de juego concretas y trabajar estrategias para el control y gestión de los partidos.

Desde la perspectiva de los participantes, el formato digital del instrumento facilita su acceso y elaboración a través de los dispositivos móviles (teléfono móvil, tableta digital y ordenador portátil). Este aspecto se valora positivamente ya que permite un acceso y proceso cómodo, rápido y sencillo. Su formato, estructura y organización se considera adecuada puesto que aborda los apartados principales a la hora de evaluar una actuación arbitral, sobre todo porque muestra sintonía con las directrices y recomendaciones prácticas que marcan las reglas de juego oficiales a nivel mundial. El tiempo precisado para cumplimentar el informe de autoevaluación fue un aspecto mejorable ya que, aunque el tiempo requerido no es elevado, necesita dedicar un esfuerzo para analizar con detenimiento la actuación después de cada partido, analizar las jugadas que han ocurrido y las decisiones tomadas. Esto implica ofrecer un tiempo razonable para llevar a cabo un análisis y valoración del desempeño adecuado, tomando conciencia de este proceso de una forma crítica y profunda (Orsmond y Merry, 2011).

La herramienta se considera útil y relevante para el equipo docente ya que le permite obtener un registro de los informes de autoevaluación realizados y llevar a cabo un seguimiento, tanto individual como colectivo, del las actuaciones realizadas. Esta información se extrae a partir de las dimensiones que incluye el instrumento. Cuando el árbitro elabora su informe tras finalizar el partido, el formador tiene el acceso a sus valoraciones con inmediatez para poder tratar los datos. Las puntuaciones otorgadas por cada participante sobre su actuación, y los aspectos destacables y con recorrido de mejora, aportan al formador datos interesantes para su posterior uso formativo.

Para realizar el informe de autoevaluación de manera unificada y adecuada es necesario facilitar orientaciones para su elaboración. Para ello, el uso de rúbricas de evaluación, que incluyen los niveles de desempeño y sus descriptores, se convierten en un recurso que complementa y enriquece la herramienta (Panadero y Romero, 2014). Permiten guiar y unificar los criterios para valorar cada una de las dimensiones. A su vez, ayuda a los formadores a preparar y utilizar los procesos de feedback dialógico (Panadero et al., 2012) en las sesiones, adaptando el enfoque de las sesiones en base a las necesidades formativas del grupo (Nicol, 2007).

De este modo, el instructor guía y acompaña a los árbitros, facilitando orientaciones, recursos y herramientas encaminadas a la mejora del desempeño de futuras actuaciones (Nicol, 2011). Se valora la integración del instrumento como recurso clave en el desarrollo de acciones formativas encaminadas a la progresión y mejora de los árbitros, provocando una autorregulación su propio aprendizaje (Panadero y Jonsson, 2013).

La información manejada por los instructores facilita la preparación del feedback antes de cada sesión de trabajo. Ayuda a seleccionar las temáticas y los contenidos a trabajar. La retroalimentación se valora como un elemento que apoya la mejora y la progresión de los árbitros (Cano, 2014; Carless, 2012, 2015; Nicol, 2011). Los materiales multimedia utilizados, vídeos sobre situaciones reales de juego (Armenteros et al., 2016), son un elemento valorado positivamente tanto por los formadores como por 
los participantes.

El enfoque pedagógico de la propuesta se ha valorado positivamente por los usuarios. Esta orientación permite generar espacios y tiempos compartidos de formación que impulsan la participación, la interacción y la colaboración entre los participantes. Estos escenarios analizan diferentes situaciones ocurridas en los partidos, generando así oportunidades para debatir y dialogar (Quinton y Smallbone, 2010), y garantizando una mejor comprensión del feedback (Hattie y Timperley, 2007; Liu y Carless, 2006; Nicol, 2010; Nicol y Macfarlane-Dick, 2006). Estos espacios invitar a compartir distintas experiencias y situaciones, las decisiones tomadas, los puntos de vista, los conceptos a considerar, dudas y sugerencias. Permiten llegar a conclusiones, teniendo en cuenta las directrices y los conceptos específicos sobre las reglas de juego. Además, se utiliza el vídeo como principal material educativo, un recurso potente desde el punto de vista didáctico (Armenteros et al., 2009). Es decir, tanto los formadores como los árbitros son parte de una comunidad de aprendizaje cuyo objetivo está en encontrar puntos de mejora, buscar recursos y adquirir herramientas para conseguir el progreso.

Para concluir, parece interesante conocer las oportunidades que presentan los distintos enfoques pedagógicos, en compañía de las prestaciones tecnológicas, con el objetivo de diseñar y desarrollar experiencias educativas en los actuales contextos de formación de árbitros y asistentes de fútbol. En esta línea, el informe de autoevaluación se convierte en un medio útil para que los árbitros lleven a cabo procesos de análisis y de reflexión críticos y constructivos sobre sus actuaciones en los terrenos de juego. De esta manera, se toma conciencia de las áreas y aspectos de mejora para buscar recursos y herramientas para lograr un avance y un progreso en futuras actuaciones arbitrales. La implementación de este tipo de acciones formativas, coherentes y contextualizadas, suponen una innovación dentro de los planes actuales de formación arbitral. Pretenden ser transferibles a otros contextos de formación arbitral en distintas modalidades deportivas. Una posible línea de trabajo está en potenciar el uso del informe de autoevaluación con el empleo de clips de vídeo, un recurso posible gracias a la creciente cobertura audiovisual que actualmente nos permite visualizar partidos de distintos deportes, que evidencien con situaciones reales el análisis de una actuación.

\section{REFERENCIAS}

Armenteros, M., y Benítez, A. J. (2011). The Integration of Educational Technology into the Training for Refereeing in Elite Football: The Use of the "Interactive Video Test". Apunts. Educación Física y Deportes(105), 12-20. https://doi.org/10.5672/apunts.2014 -0983.es.(2011/3).105.01

Armenteros, M., Benítez, A. J., y Curca, D. G. (2011). El trivial interactivo como recurso educativo para el aprendizaje de las reglas de juego del fútbol. Píxel-Bit. Revista de Medios y Educación, 38, 35-48. Descargado de https://recyt.fecyt.es/index.php/pixel/ article/view/61415

Armenteros, M., Benítez, A. J., y Curca, D. G. (2013). Interactive Trivia of Laws of the Game as a Resource for Training FIFA Football Referees. En H. H. Yang y S. Wang (Eds.), Cases on Formal and Informal E-Learning Environments: Opportunities and Practices (pp. 200-214). IGI Global. https://doi.org//10.4018/978-1-4666-1930-2

Armenteros, M., Benítez, A. J., Fernández, M., la Vega, R. D., Sillero-Quintana, M., y SánchezCid, M. (2019). Collaborative learning methods and multimedia tools for the education and training of instructors: the case of FIFA referee technical instructors. International fournal of Information and Learning Technology, 36(5), 395-409. Descargado de https:// www.emerald.com/insight/content/doi/10.1108/IJILT-07-2017-0061/full/html

Armenteros, M., Benítez, A. J., Flores, R., Sillero-Quintana, M., Sánchez-Cid, M., y Simón, J. A. (2018). The training of soccer assistant referees beyond on-field experience: the use of the Interactive Video Test. International Journal of Computer Science in Sport, 17(2), 
163-174. https://doi.org/10.2478/ijcss-2018-0009

Armenteros, M., Benítez, A. J., y Sillero, M. (2009). Advantages of Applying Video to Interactive Auto-Evaluation: A Model used for the Training of Referees and Instructors of the Fédération Internationale of Football Association (FIFA) and the Union of European Football Associations (UEFA). Proceeding of Internet and Multimedia Systems and Applications (pp. 13-15).

Armenteros, M., Domínguez, A., Fernández, M., y Benítez, A. J. (2016). The Video Database for Teaching and Learning in Football Refereeing. World Academy of Science, Engineering and Technology, International fournal of Social, Behavioral, Educational, Economic, Business and Industrial Engineering, 10(9), 3134-3139. https://doi.org/ 10.5281/zenodo.1126517

Armenteros, M., Liaw, S. S., Fernández, M., Flores-Díaz, R., y Arteaga-Sánchez, R. (2013). Surveying FIFA instructors' behavioral intention toward the Multimedia Teaching Materials. Computers \& Education, 61, 91-104. https://doi.org/10.1016/j.compedu.2012 .09 .010

Armenteros, M., Liaw, S. S., Sánchez-Franco, M. J., Fernández, M., y Arteaga-Sánchez, R. (2017). Analysis of FIFA referees and assistant referees' motivational factors towards the Multimedia Teaching Materials. Education and Information Technologies, 22, 841872. https://doi.org/10.1007/s10639-015-9460-y

Arribas, M. C. M. (2004). Diseño y validación de cuestionarios. Matronas Profesión, 5(17), 23-29.

Cano, E. (2014). Análisis de las investigaciones sobre feedback: aportes para su mejora en el marco del EEES. Bordón. Revista de Pedagogía, 66(4), 9-24. Descargado de https:// recyt.fecyt.es/index.php/BORDON/article/view/Bordon.2014.66402

Cardoso, S. M., Travassos, B., y Oliveira, E. P. (2020). Desenvolvimento de carreiras e perceção de fatores para a excelência do árbitro de futebol em Portugal. Retos(37), 694-701. https://doi.org/10.47197/retos.v37i37.74350

Carless, D. (2012). Trust and its role in facilitating dialogic feedback. En D. Boud y E. Molloy (Eds.), Feedback in Higher and Professional Education (pp. 90-103). London: Routledge.

Carless, D. (2015). Excellence in University Assessment: Learning from award-winning practice. Londres \& Nueva York: Routledge.

CTA. (2018). Guía para Delegados de Partido e Informadores de 2aivisión B. Descargado de https://bit.ly/3kgruyt

Dosseville, F., y Laborde, S. (2015). Introduction to the special issue: Officials in sports. Movement \& Sport Sciences - Science \& Motricité, 87, 3-10. https://doi.org/10.1051/sm/ 2015006

Escobar-Pérez, J., y Cuervo-Martínez, A. (2008). Validez de contenido y juicio de expertos: Una aproximación a su utilización. Avances En Medición, 6(1), 27-36. Descargado de http://www.humanas.unal.edu.co/psicometria/files/7113/8574/5708/Articulo3_Juicio de_expertos_27-36.pdf

Gibbs, G., y Simpson, C. (2004). Conditions under which assessment supports students' learning. Learning and Teaching in Higher Education, 1(1), 1-31. Descargado de http:// eprints.glos.ac.uk/id/eprint/3609

González-Oya, J. (2005). Reflexiones sobre la formación del árbitro: El papel de la psicopedagogía en el sistema educativo arbitral. Revista Galego-Portuguesa De Psicoloxía e Educación, 10(12), 187-194. Descargado de https://core.ac.uk/download/ pdf/61900599.pdf

Hancock, D. J., Rix-Lièvre, G., y Côté, J. (2015). Citation network analysis of research on sport officials: a lack of interconnectivity. International Review of Sport and Exercise Psychology, 8(1), 95-105. https://doi.org/10.1080/1750984X.2015.1022202

Hattie, J., y Timperley, H. (2007). The power of feedback. Review of Educational Research, 77(1), 81-112. https://doi.org/10.3102/003465430298487

IFAB. (2019). Reglas de fuego 2019-2020. Descargado de https://bit.ly/2GRxGzq 
Liu, N. F., y Carless, D. (2006). Peer feedback: The learning element of peer assessment. Teaching in Higher Education, 11(3), 279-290. https://doi.org/10.1080/ 13562510600680582

Nicol, D. (2007). Principles of good assessment and feedback: Theory and practice. REAP International Online Conference on Assessment Design for Learner Responsibility.

Nicol, D. (2010). From monologue to dialogue: Improving written feedback processes in mass higher education. Assessment \& Evaluation in Higher Education, 35(5), 501-17. https://doi.org/10.1080/02602931003786559

Nicol, D. (2011). Good designs for written feedback for students. En M. Svinicki y W. J. McKeachie (Eds.), Teaching tips: Strategies, Research and Theories for College and University Teachers (pp. 108-124). Belmont, USA: Wadsworth Cengage Learning.

Nicol, D., y Macfarlane-Dick, D. (2006). Formative assessment and self-regulated learning: A model and seven principles of good feedback practice. Studies in Higher Education, 31, 199-218. https://doi.org/10.1080/03075070600572090

Orsmond, P., Maw, S. J., Park, J. R., Gomez, S., y Crook, A. C. (2013). Moving feedback forward: theory to practice. Assessment \& Evaluation in Higher Education, 38(2), 240252. https://doi.org/10.1080/02602938.2011.625472

Orsmond, P., y Merry, S. (2011). Feedback alignment: Effective and ineffective links between tutors' and students' understanding of coursework feedback. Assessment \& Evaluation in Higher Education, 36(2), 125-36. https://doi.org/10.1080/02602930903201651

Panadero, E., Alonso-Tapia, J., y Huertas, J. A. (2012). Rubrics and self-assessment scripts effects on self-regulation, learning and self-efficacy in secondary education. Learning and Individual Differences, 22(6), 806-813. https://doi.org/10.1016/j.lindif.2012.04.007

Panadero, E., y Jonsson, A. (2013). The use of scoring rubrics for formative assessment purposes revisited: A review. Educational Research Review, 9, 129-144.

Panadero, E., y Romero, M. (2014). To rubric or not to rubric? The effects of self-assessment on self-regulation, performance and self-efficacy. Assessment in Education: Principles, Policy \& Practice, 21(2), 133-148. https://doi.org/10.1080/0969594X.2013.877872

Price, M., Handley, K., Millar, J., y O’Donovan, B. (2010). Feedback: all that effort, but what is the effect? Assessment \& Evaluation in Higher Education, 35(3), 277-289. https:// doi.org/10.1080/02602930903541007

Quinton, S., y Smallbone, T. (2010). Feeding forward: using feedback to promote student reflection and learning- a teacher model. Innovations in Education and Teaching International, 47(1), 125-135.

Recio-Moreno, D., Feliz-Murias, T., y Elorza-Barbajero, J. (2020). La formación de árbitros y asistentes de fútbol desde el enfoque flipped learning. Retos(39), 794-804. https:// doi.org/10.47197/retos.v0i39.78222

Samuel, R. D., Galily, Y., y Tenenbaum, G. (2017). Who are you, ref? Defining the soccer referee's career using a change-based perspective. International fournal of Sport and Exercise Psychology, 15(2), 118-130. https://doi.org/10.1080/1612197X.2015.1079792

Serrano, G. P. (1994). Investigación cualitativa. Retos e interrogantes. I. Métodos. Madrid: La Muralla.

Stake, R. E. (2005). Investigación con estudio de casos. Madrid: Morata.

UEFA. (2019). Refereeing Assistance Programme 2019. Descargado de https://bit.ly/3lnpkOX 